\title{
Probing a Finite Unified Theory with Reduced Couplings at Future Colliders
}

\author{
S. Heinemeyer, ${ }^{a, b, c}$ J. Kalinowski, ${ }^{d}$ W. Kotlarski, ${ }^{e}$ M. Mondragón, ${ }^{f}$ G. Patellis, ${ }^{g, *}$ N. \\ Tracas $^{g}$ and G. Zoupanos ${ }^{g, h, i}$ \\ ${ }^{a}$ Instituto de Física Teórica (UAM/CSIC), Universidad Autónoma de Madrid, \\ Cantoblanco, 28049 Madrid, Spain \\ ${ }^{b}$ Campus of International Excellence UAM+CSIC, Cantoblanco, 28049 Madrid, Spain \\ ${ }^{c}$ Instituto de Física de Cantabria (CSIC-UC), E-39005 Santander, Spain \\ ${ }^{d}$ University of Warsaw - Faculty of Physics, ul. Pasteura 5, 02-093 Warsaw, Poland \\ ${ }^{e}$ Technische Universität Dresden - Institut für Kern- und Teilchenphysik (IKTP), 01069 Dresden, Germany \\ ${ }^{f}$ Instituto de Física, Universidad Nacional Autónoma de México, \\ A.P. 20-364, CDMX 01000 México \\ ${ }^{g}$ Physics Department, Nat. Technical University, 15780 Zografou, Athens, Greece \\ ${ }^{h}$ Max-Planck Institut für Physik, Föhringer Ring 6, D-80805 München, Germany \\ ${ }^{i}$ Theoretical Physics Department, CERN, Geneva, Switzerland \\ E-mail: Sven.Heinemeyer@cern.ch, kalino@fuw.edu.pl, \\ wojciech.kotlarski@tu-dresden.de, myriam@fisica.unam.mx, \\ patellis@central.ntua.gr,ntrac@central.ntua.gr, George.Zoupanos@cern.ch
}

The search for relations among parameters that are renormalization group invariant to all orders in perturbation theory constitutes the basis of the reduction of couplings idea. Reduction of couplings can be achieved in $N=1$ Grand Unified Theories, few of which can become even all-loop finite. We review the basic idea and a resulting theory in which successful reduction of couplings has been achieved, namely the all-loop finite $N=1$ supersymmetric $S U(5)$ model. We present three benchmark scenarios and investigate their observability at existing and future hadron colliders. The supersymmetric spectrum is found to be beyond the reach of the $14 \mathrm{TeV}$ HL-LHC. In turn, it is found that large parts of the predicted spectrum can be tested at the $100 \mathrm{TeV}$ FCC-hh, but the higher mass regions remain out of reach.

\footnotetext{
*** The European Physical Society Conference on High Energy Physics (EPS-HEP2021), ***

*** 26-30 July $2021 * * *$

*** Online conference, jointly organized by Universität Hamburg and the research center DESY ***
}

\footnotetext{
${ }^{*}$ Speaker
} 


\section{Introduction}

The reduction of couplings idea [1] is a promising method which relates seemingly independent parameters to a single coupling. The method requires the original theory to be renormalizable and the resulting relations among parameters to be valid at all energy scales, that is Renormalization Group Invariant (RGI). A next step, after the introduction of a new symmetry through a Grand Unified Theory (GUT), in order to achieve reduction of free parameters of the Standard Model (SM), is the relation of the gauge sector to the Yukawa sector. Then RGI relations (even such that can guarantee all order finiteness of a theory) are set between the unification scale and the Planck scale. Since supersymmetry (SUSY) seems to be a crucial ingredient for the reduction of couplings technique to render viable phenomenology, we have to include a supersymmetry breaking sector (SSB). Significant progress in this direction has lead to complete all-loop finite models, i.e. including the SSB sector. Past applications of the above method [2, 3] have predicted the top quark mass one year before its experimental measurement. Furthermore, the all-loop finite $N=1 S U(5)$ model [4] has also given a prediction for the Higgs mass compatible with the experimental results and predicts a heavy SUSY mass spectrum, consistent with the experimental non-observation of these particles. The full analysis of the most successful models can be found in recent works [5, 6].

In this article we address the question to what extent the reduction of couplings idea can be tested at HL-LHC and FCC. To this end we propose three benchmark points for the Finite $N=1 S U(5)$ model and, using the SSB parameters as input in each benchmark we calculate the corresponding Higgs and SUSY spectra. Then we compute the expected production cross sections at the $14 \mathrm{TeV}$ (HL-)LHC and the $100 \mathrm{TeV}$ FCC-hh and investigate which production channels can be observed.

\section{Theoretical Basis}

Here we will briefly review the core idea of the reduction of couplings method. The target is to single out a basic parameter, where all other parameters can be expressed in terms of this one through RGI relations. Such a relation has, in general, the form $\Phi\left(g_{1}, \cdots, g_{A}\right)=$ const. which should satisfy the following partial differential equation (PDE)

$$
\mu \frac{d \Phi}{d \mu}=\vec{\nabla} \Phi \cdot \vec{\beta}=\sum_{a=1}^{A} \beta_{a} \frac{\partial \Phi}{\partial g_{a}}=0,
$$

where $\beta_{a}$ is the $\beta$-functions of $g_{a}$. The above PDE is equivalent to the following set of ordinary differential equations (ODEs), which are called Reduction Equations (REs) [1, 7],

$$
\beta_{g} \frac{d g_{a}}{d g}=\beta_{a}, a=1, \cdots, A-1
$$

where now $g$ and $\beta_{g}$ are the primary coupling and its corresponding $\beta$-function. The crucial demand is that the above REs admit power series solutions

$$
g_{a}=\sum_{n} \rho_{a}^{(n)} g^{2 n+1},
$$


which preserve perturbative renormalizability. Without this requirement, we just trade each "dependent" coupling for an integration constant. It is very important to point out that the uniqueness of such a solution can be already decided at the one-loop level $[1,7]$. Concerning the reduction scheme for massive parameters, a number of conditions is required. Nevertheless, progress has been achieved, starting from [8], and finally we can introduce mass parameters and couplings carrying mass dimension $[9,10]$ in the same way as dimensionless couplings.

Consider an $N=1$ gauge theory, chiral and anomaly free, where $G$ is the gauge group and $g$ the associated gauge coupling. The one-loop $\beta$-function of the gauge coupling $[11,12]$ and the one-loop anomalous dimension $\gamma^{(1)} \underset{j}{i}$ of a chiral superfield are

$\beta_{g}^{(1)}=\frac{d g}{d t}=\frac{g^{3}}{16 \pi^{2}}\left[\sum_{i} T\left(R_{i}\right)-3 C_{2}(G)\right], \quad \gamma_{j}^{(1) i}=\frac{1}{32 \pi^{2}}\left[C^{i k l} C_{j k l}-2 g^{2} C_{2}\left(R_{i}\right) \delta_{j}^{i}\right]$,

where $T\left(R_{i}\right)$ is the Dynkin index of the rep $R_{i}$ where the matter fields belong and $C_{2}(G)$ is the quadratic Casimir operator of the adjoint rep $G$ and $C_{i j k}$ are the Yukawa couplings. Demanding the vanishing of all one-loop $\beta$-functions, we get the relations

$$
\sum_{i} T\left(R_{i}\right)=3 C_{2}(G), \quad C^{i k l} C_{j k l}=2 \delta_{j}^{i} g^{2} C_{2}\left(R_{i}\right)
$$

The finiteness conditions for an $N=1$ supersymmetric theory with $S U(N)$ associated group is found in [13]. Conditions (5) are necessary and sufficient to ensure two-loop finiteness [11, 14, 15]. The requirement of one-loop finiteness in softly broken SUSY theories demands additional constraints among the soft terms of the SSB sector [16], while, once more, these one-loop requirements assure two-loop finiteness, too [17].

The non-trivial point is that the relations among couplings (gauge and Yukawa) which are imposed by the conditions (5) should hold at any energy scale. The necessary and sufficient condition is to require that such relations are solutions to the REs

$$
\beta_{g} \frac{d C_{i j k}}{d g}=\beta_{i j k}
$$

holding at all orders. A theorem which points down which are the necessary and sufficient conditions in order for an $N=1$ SUSY theory to be all-loop finite can be found in [18]. A comprehensive review of the above can be found in [19] and [20].

\section{The Finite $\mathbf{N}=1$ Supersymmetric SU(5) Model}

In this all-loop finite $S U(5)$ gauge theory the reduction of couplings is restricted to the third generation. An older examination of this specific Finite Unified Theory (FUT) was shown to be in agreement with the experimental constraints at the time [4] and has predicted, almost five years before its discovery, the light Higgs mass in the correct range. The particle content of the model has three $(\overline{\mathbf{5}}+\mathbf{1 0})$ supermultiplets for the three generations of leptons and quarks, while the Higgs sector consists of four supermultiplets $(\overline{\mathbf{5}}+\mathbf{5})$ and one $\mathbf{2 4}$. The finite $S U(5)$ group is broken to the MSSM, which of course in no longer a finite theory [2, 3, 21]. 
When $S U(5)$ breaks down to the MSSM, a suitable rotation in the Higgs sector [2, 21-24], permits only a pair of Higgs doublets (coupled mostly to the third family) to remain light and acquire vev's. Avoiding fast proton decay is achieved with the usual doublet-triplet splitting. Therefore, below the GUT scale we get the MSSM where the third generation is given by the finiteness conditions while the first two remain unrestricted.

Conditions set by finiteness do not restrict the renormalization properties at low energies, so we are left with boundary conditions on the gauge and Yukawa couplings (plus the boundary conditions that occur in the soft sector) at $M_{\mathrm{GUT}}$. The quark masses $m_{b}\left(M_{Z}\right)$ and $m_{t}$ are predicted within $2 \sigma$ and $3 \sigma$ uncertainty, respectively, of their experimental values. The only phenomenologically viable option is to consider $\mu<0$, as shown in [5, 19, 25-28]. The light Higgs boson mass is predicted within $1 \sigma$ of its experimental value, while the (point-by-point) theoretical uncertainty [29] (calculated with FeynHiggs [30]) drops significantly (w.r.t. past analyses) to $0.65-0.70 \mathrm{GeV}$ (see [5] for details).

As explained in more detail in [6], the three benchmarks chosen (for the purposes of collider phenomenology) feature the LSP above $2100 \mathrm{GeV}, 2400 \mathrm{GeV}$ and $2900 \mathrm{GeV}$, respectively. The resulting masses that are relevant to our analysis were generated by SPheno 4.0.4 [31] and are listed in Table 1 for each benchmark (with the corresponding $\tan \beta$ ).

\begin{tabular}{|l|r|r|r|r|r|r|r|r|r|r|}
\hline & $\tan \beta$ & $M_{A, H}$ & $M_{H^{ \pm}}$ & $M_{\tilde{g}}$ & $M_{\tilde{\chi}_{1}^{0}}$ & $M_{\tilde{\chi}_{2}^{0}}$ & $M_{\tilde{\chi}_{3}^{0}}$ & $M_{\tilde{\chi}_{4}^{0}}$ & $M_{\tilde{\chi}_{1}^{ \pm}}$ & $M_{\tilde{\chi}_{2}^{ \pm}}$ \\
\hline FUTSU5-1 & 49.9 & 5.688 & 5.688 & 8.966 & 2.103 & 3.917 & 4.829 & 4.832 & 3.917 & 4.833 \\
\hline FUTSU5-2 & 50.1 & 7.039 & 7.086 & 10.380 & 2.476 & 4.592 & 5.515 & 5.518 & 4.592 & 5.519 \\
\hline FUTSU5-3 & 49.9 & 16.382 & 16.401 & 12.210 & 2.972 & 5.484 & 6.688 & 6.691 & 5.484 & 6.691 \\
\hline & $M_{\tilde{e}_{1,2}}$ & $M_{\tilde{\nu}_{1,2}}$ & $M_{\tilde{\tau}}$ & $M_{\tilde{\nu}_{\tau}}$ & $M_{\tilde{d}_{1,2}}$ & $M_{\tilde{u}_{1,2}}$ & $M_{\tilde{b}_{1}}$ & $M_{\tilde{b}_{2}}$ & $M_{\tilde{t}_{1}}$ & $M_{\tilde{t}_{2}}$ \\
\hline FUTSU5-1 & 3.102 & 3.907 & 2.205 & 3.137 & 7.839 & 7.888 & 6.102 & 6.817 & 6.099 & 6.821 \\
\hline FUTSU5-2 & 3.623 & 4.566 & 2.517 & 3.768 & 9.059 & 9.119 & 7.113 & 7.877 & 7.032 & 7.881 \\
\hline FUTSU5-3 & 4.334 & 5.418 & 3.426 & 3.834 & 10.635 & 10.699 & 8.000 & 9.387 & 8.401 & 9.390 \\
\hline
\end{tabular}

Table 1: Masses for the three benchmarks of the Finite $N=1 S U(5)$ (in TeV) [6].

At $14 \mathrm{TeV}$ HL-LHC none of the Finite $S U(5)$ scenarios listed above has a SUSY production cross section above $0.01 \mathrm{fb}$, and thus will most probably remain unobservable [32]. The discovery prospects for the heavy Higgs-boson spectrum is significantly better at the FCC-hh [33]. Theoretical analyses $[33,34]$ have shown that for large $\tan \beta$ heavy Higgs mass scales up to $\sim 8 \mathrm{TeV}$ could be accessible. Since in this model we have $\tan \beta \sim 50$, the first two benchmark points are well within the reach of the FCC-hh (as explained in [6]). The third point, however, where $M_{A} \sim 16 \mathrm{TeV}$, will be far outside the reach of the collider. At $100 \mathrm{TeV}$ we have in principle production of SUSY particles in pairs, although their production cross section is at the few fb level. This is a result of the heavy spectrum of the model. Comparing our benchmark predictions with the simplified model limits of [35], we have found that the lighter stop might be accessible in FUTSU5-1 (see [6]). For the squarks of the first two generations there are better prospects. All benchmarks could be tested at the $2 \sigma$ level, but no discovery at the $5 \sigma$ can be expected and the same holds for the gluino. The expected production cross sections for various final states and a more exhaustive discussion can be found in [6]. 


\section{Conclusions}

The reduction of couplings scheme consists in searching for RGE relations among parameters of a renormalizable theory that hold to all orders in perturbation theory. In certain $N=1$ theories the reduction of couplings idea and the concept of finiteness are theoretically realised and develop powerful tools able to increase the predictivity of these theories. In the present article we turned to the question of testing experimentally the idea of reduction of couplings. A finite model based on the $S U(5)$ gauge theory is reviewed.

The model is found to be in comfortable agreement with LHC measurements and searches, while it predicts a relatively heavy spectrum which evades largely the detection in the HLLHC. Concerning the accessibility of the SUSY and heavy Higgs spectra at the FCC-hh with $\sqrt{s}=100 \mathrm{TeV}$, we found that the lower parts of the parameter space will be testable at the $2 \sigma$ level, with only an even smaller part discoverable at the $5 \sigma$ level. However, the heavier parts of the SUSY spectrum will remain elusive even at the FCC-hh.

This work has been supported by the HARMONIA project, National Science Center - Poland, contract UMO-2015/18/M/ST2/00518 and by the Basic Research Programme, PEVE2020 of the National Technical University of Athens, Greece. The work of M.M. is supported by a DGAPAUNAM grant PAPIIT IN109321.

\section{References}

[1] W. Zimmermann, Commun. Math. Phys. 97, 211 (1985).

[2] D. Kapetanakis, M. Mondragon, and G. Zoupanos, Z. Phys. C60, 181 (1993), arXiv:hep$\mathrm{ph} / 9210218$.

[3] J. Kubo, M. Mondragon, and G. Zoupanos, Nucl. Phys. B424, 291 (1994).

[4] S. Heinemeyer, M. Mondragon, and G. Zoupanos, JHEP 07, 135 (2008), arXiv:0712.3630.

[5] S. Heinemeyer, M. Mondragón, G. Patellis, N. Tracas, and G. Zoupanos, Fortsch. Phys. 68, 2000028 (2020), arXiv:2002.10983.

[6] S. Heinemeyer et al., Eur. Phys. J. C81, 185 (2021), arXiv:2011.07900.

[7] R. Oehme and W. Zimmermann, Commun. Math. Phys. 97, 569 (1985).

[8] J. Kubo, M. Mondragon, and G. Zoupanos, Phys. Lett. B389, 523 (1996), arXiv:hep$\mathrm{ph} / 9609218$.

[9] P. Breitenlohner and D. Maison, Commun. Math. Phys. 219, 179 (2001).

[10] W. Zimmermann, Commun.Math.Phys. 219, 221 (2001).

[11] A. Parkes and P. C. West, Phys. Lett. B138, 99 (1984).

[12] D. R. T. Jones and A. J. Parkes, Phys. Lett. B160, 267 (1985). 
[13] S. Rajpoot and J. G. Taylor, Phys. Lett. B147, 91 (1984).

[14] P. C. West, Phys. Lett. B137, 371 (1984).

[15] D. R. T. Jones and L. Mezincescu, Phys. Lett. B138, 293 (1984).

[16] D. R. T. Jones, L. Mezincescu, and Y. P. Yao, Phys. Lett. B148, 317 (1984).

[17] I. Jack and D. R. T. Jones, Phys. Lett. B333, 372 (1994), hep-ph/9405233.

[18] C. Lucchesi, O. Piguet, and K. Sibold, Phys. Lett. B201, 241 (1988).

[19] S. Heinemeyer, M. Mondragón, N. Tracas, and G. Zoupanos, Phys. Rept. 814, 1 (2019), arXiv:1904.00410.

[20] G. Patellis, PhD thesis, Natl. Tech. U., Athens, 2020, arXiv:2102.01476.

[21] M. Mondragon and G. Zoupanos, Nucl. Phys. Proc. Suppl. 37C, 98 (1995).

[22] J. Leon, J. Perez-Mercader, M. Quiros, and J. Ramirez-Mittelbrunn, Phys. Lett. B156, 66 (1985).

[23] S. Hamidi and J. H. Schwarz, Phys. Lett. B147, 301 (1984).

[24] D. R. T. Jones and S. Raby, Phys. Lett. B143, 137 (1984).

[25] S. Heinemeyer, M. Mondragon, and G. Zoupanos, Phys.Lett. B718, 1430 (2013), arXiv:1211.3765.

[26] S. Heinemeyer, M. Mondragon, and G. Zoupanos, Phys.Part.Nucl. 44, 299 (2013).

[27] S. Heinemeyer, M. Mondragón, G. Patellis, N. Tracas, and G. Zoupanos, Symmetry 10, 62 (2018), arXiv:1802.04666.

[28] S. Heinemeyer, M. Mondragón, N. D. Tracas, and G. Zoupanos, Ann. U. Craiova Phys. 24, 56 (2014).

[29] H. Bahl, S. Heinemeyer, W. Hollik, and G. Weiglein, Eur. Phys. J. C80, 497 (2020), arXiv:1912.04199.

[30] H. Bahl et al., Comput. Phys. Commun. 249, 107099 (2020), arXiv:1811.09073.

[31] W. Porod and F. Staub, Comput. Phys. Commun. 183, 2458 (2012), arXiv:1104.1573.

[32] M. Cepeda et al., CERN Yellow Rep. Monogr. 7, 221 (2019), arXiv:1902.00134.

[33] J. Hajer, Y.-Y. Li, T. Liu, and J. F. H. Shiu, JHEP 11, 124 (2015), arXiv:1504.07617.

[34] N. Craig, J. Hajer, Y.-Y. Li, T. Liu, and H. Zhang, JHEP 01, 018 (2017), arXiv:1605.08744.

[35] T. Golling et al., (2016), arXiv:1606.00947. 